\title{
Efecto de la etapa de levante sobre la producción y reproducción en codornices japónicas (Coturnix coturnix japonica) de postura
}

\author{
Effect of the rearing stage on production and reproduction in laying Japanese \\ quail (Coturnix coturnix japonica)
}

\author{
Fritz Trillo Z..$^{1,4}$, Pedro Ciriaco C. ${ }^{1}$, Lincol Tafur C. ${ }^{2}$, Virginia Rivadeneira ${ }^{3}$, \\ Nadia Fuentes N. ${ }^{3}$, Jimny Nuñez D. ${ }^{3}$
}

\section{Resumen}

El objetivo de la investigación fue evaluar el rendimiento productivo de codornices (Coturnix coturnix japonica) de postura. El estudio se realizó en la granja de aves de la Universidad Nacional Agraria La Molina en Lima, Perú. Se utilizaron 1296 codornices distribuidas en 96 hembras y 48 machos de tres edades (45, 52 y 59 días) con 130, 140 y $150 \mathrm{~g}$ de peso al inicio de la postura, respectivamente. Se empleó un diseño de bloques aleatorios con arreglo factorial $3^{2}$ teniendo como factores edad y peso al inicio de postura y como bloque a las semanas de postura $(8,11$ y 17). Las mejores conversiones alimenticias fueron de $3.67 \pm 0.36$ y de $3.68 \pm 0.35$, sin diferencias estadísticas entre ellas, y se obtuvieron cuando las aves iniciaron su postura con pesos de 130 y $140 \mathrm{~g}$, respectivamente. La mayor masa de huevos ( $10.72 \pm 0.41 \mathrm{~g} /$ día/ave $)$ se obtuvo cuando las aves iniciaron su postura con $150 \mathrm{~g}$ de peso vivo.

Palabras clave: conversión alimenticia, masa de huevo, peso vivo, postura de huevos

\footnotetext{
${ }^{1}$ Departamento de Producción Animal, Facultad de Zootecnia, Universidad Nacional Agraria La Molina, Lima, Perú

${ }^{2}$ Escuela Académico Profesional de Ingeniería Zootecnia, Facultad de Ingeniería en Ciencias Pecuarias, Universidad Nacional de Cajamarca, Lima, Perú

${ }^{3}$ Departamento de Producción Animal, Facultad de Medicina Veterinaria, Universidad Nacional Mayor de San Marcos, Lima, Perú

${ }^{4}$ E-mail: ftrillo@lamolina.edu.pe
}

Recibido: 15 de febrero de 2021

Aceptado para publicación: 31 de agosto de 2021

Publicado: 27 de octubre de 2021

CLos autores. Este artículo es publicado por la Rev Inv Vet Perú de la Facultad de Medicina Veterinaria, Universidad Nacional Mayor de San Marcos. Este es un artículo de acceso abierto, distribuido bajo los términos de la licencia Creative Commons Atribución 4.0 Internacional (CC BY 4.0) [https:// creativecommons.org/licenses/by/4.0/deed.es] que permite el uso, distribución y reproducción en cualquier medio, siempre que la obra original sea debidamente citada de su fuente original 
The aim of this study was to evaluate the productive performance of laying quails (Coturnix coturnix japonica). The study was carried out at the poultry farm of the La Molina National Agrarian University in Lima, Peru. In total, 1296 quail distributed in 96 females and 48 males of three ages (45, 52 and 59 days) with 130, 140 and $150 \mathrm{~g}$ of body weight, respectively, at the beginning of the laying were used. A random block design with $\mathrm{a}^{2}$ factorial arrangement was used, taking as factors age and weight at the beginning of laying and as a block the weeks of laying $(8,11$ and 17). The highest egg mass (10.72 $\pm 0.41 \mathrm{~g} /$ day/bird) was obtained when the quails started laying with $150 \mathrm{~g}$ of live weight.

Key words: feed conversion, egg mass, body weight, egg laying

\section{INTRODUCCIÓN}

Debido a la alta demanda de proteína animal para la seguridad alimentaria, la avicultura juega un rol importante como fuente económica, además de ser un activo de capital para los productores avícolas (FAO, 2013). Actualmente se fomenta la crianza familiar y comercial de algunas especies poco utilizadas como la codorniz, la cual se adapta fácilmente a la crianza familiar y comercial. El peso de la codorniz doméstica se ha logrado incrementar desde $45 \%$ hasta más de $250 \%$, dependiendo del tipo de crianza; siendo un aspecto para resaltar la influencia de la domesticación sobre su capacidad de puesta de huevos (Lukanov y Pavlova, 2020). La codorniz de mayor distribución mundial es la especie Coturnix coturnix japonica, la que tiene como ventaja ser una reserva importante de carne y huevos, con alto contenido proteico y bajos niveles de grasa (Da Cunha, 2009), siendo su composición química similar a la carne de pollo (Cori et al., 2014).

La Coturnix coturnix japonica es rústica, se cría en jaulas pequeñas y su alimentación y manejo son baratos. Además, tiene una mayor resistencia a las enfermedades comunes de las aves (Randall y Bolla, 2006).
Son aves precoces, pues alcanzan la madurez sexual a las seis semanas y la mayoría están en plena producción de huevos a los 50 días de edad; sin embargo, la fertilidad, mortalidad y eclosión en las aves están directamente relacionadas al potencial genético y a factores ambientales (Kuurman et al., 2002).

La productividad de un sistema de crianza en aves se puede evaluar mediante el recuento de huevos de calidad producidos y el número de pollitos eclosionados de estos huevos (Sahin et al., 2009). Existen diversos factores que afectan la eclosión y el rendimiento posterior a la eclosión, muchos de ellos dependientes del ciclo de postura. La tasa de eclosión se puede asociar al peso del huevo, siendo los de mayor eclosión aquellos huevos con pesos mayores a $10 \mathrm{~g}$ (Galíndez et al., 2009).

La explotación de codornices en el Perú se viene incrementando en forma considerable debido a los beneficios que brindan; sin embargo, se conoce poco sobre el rendimiento productivo de estas aves de postura. En consecuencia, el objetivo de esta investigación fue comparar el rendimiento productivo de Coturnix coturnix japonica a diferentes edades y pesos al inicio del empadre. 


\section{Materiales y Métodos}

\section{Ubicación del Experimento}

El estudio se realizó en la granja de aves de la Universidad Nacional Agraria La Molina (UNALM), ubicado la ciudad de Lima. Durante el estudio, la temperatura ambiental fue de $18.5^{\circ} \mathrm{C}$ y la humedad relativa de $88 \%$.

\section{Equipos e Instalaciones}

Se utilizaron nueve baterías metálicas para codornices de seis pisos, equipadas con comederos lineales galvanizados y bebederos tipo copa en la parte frontal. Además, tuvieron su bandeja estercolera de plástico. Las jaulas medían $61 \mathrm{~cm}$ (largo), $75 \mathrm{~cm}$ (ancho), $22 \mathrm{~cm}$ (alto) con un área de $0.20 \mathrm{~m}^{2}$.

Los huevos para la incubación fueron seleccionados por tamaño, calidad de cáscara, suciedad, pigmentación verdosa, sin pigmentación y fárfara. La calidad se evaluó semanalmente en huevos recolectados del día de manera aleatoria.

\section{Aves e Incubación}

Se utilizaron 1296 codornices procedentes de tres lotes de diferente edad en reproducción. Aleatoriamente se eligieron 96 hembras y 48 machos (1:3) de tres edades ( 45 , 52 y 59 días) con 130,140 y $150 \mathrm{~g}$ de peso al inicio de postura, respectivamente, por tratamiento en las baterías (Cuadro 1). Las aves estuvieron con buen estado de salud y eran físicamente uniformes.

La dieta fue elaborada en la Planta de Alimentos de la UNALM, y contenía 2846 $\mathrm{kcal} / \mathrm{kg}$ de energía metabolizable, $19.32 \%$ de proteína cruda, $1.14 \%$ de lisina, $0.52 \%$ de metionina, $3.24 \%$ de calcio y $0.27 \%$ de fósforo disponible. La ración fue de $25 \mathrm{~g} / \mathrm{ave} /$ día, entre la semana 7 y 17 de edad.
Cuadro 1. Tratamientos en arreglo factorial de $3^{2}$

\begin{tabular}{ccc}
\hline Tratamiento & $\begin{array}{c}\text { Edad } \\
\text { (días) }\end{array}$ & $\begin{array}{c}\text { Peso vivo } \\
\text { (g) }\end{array}$ \\
\hline T1 & 45 & 130 \\
T2 & & 140 \\
T3 & & 150 \\
T4 & 52 & 130 \\
T5 & & 140 \\
T6 & & 150 \\
T7 & 59 & 130 \\
T8 & & 140 \\
T9 & & 150 \\
\hline
\end{tabular}

Los huevos se recolectaron a primera hora del día, fueron rotulados y colocados en bandejas con el polo agudo hacia abajo, luego fueron desinfectados con Tek Trol (yodóforo desinfectante-detergente sintético) según las indicaciones del producto. La incubación se realizó utilizando la incubadora/nacedora Robbins ${ }^{\circledR}$ con capacidad para 19800 huevos. Los parámetros de incubación fueron: $37.5{ }^{\circ} \mathrm{C}$ de temperatura y $60 \%$ de humedad relativa. Los huevos fueron incubados durante 18 días, separándose en dos etapas (14 días en incubadora y 4 días en nacedora).

\section{Mediciones}

Se tomaron registros de la producción y calidad del huevo. Los pesos se registraron utilizando una balanza digital (Ohaus ${ }^{\circledR}$, Ranger 3000) con capacidad de 3000 g y precisión de $0.1 \mathrm{~g}$. Todos los pesos fueron expresados en gramos. 
Los parámetros productivos evaluados fueron:

- $\quad$ Número de huevos por ave $\left(\mathrm{NHD}=\mathrm{N}^{\circ}\right.$ de huevos puestos / $\left(\mathrm{N}^{\circ}\right.$ reproductoras * días totales))

- $\quad$ Tasa de postura $\left(\mathrm{PP}=\left(\mathrm{N}^{\circ}\right.\right.$ huevos puestos / $\mathrm{N}^{\circ}$ reproductoras) $* 100$ )

- $\quad$ Peso promedio de huevos (PHP = Peso de los huevos día / $\mathrm{N}^{\circ}$ huevos puestos día)

- Masa de huevos $\left(\mathrm{MH}=\mathrm{N}^{\circ}\right.$ huevos / tiempo / ave * PHP)

- Consumo de alimento por ave por día $\left(\mathrm{CAD}=\right.$ Consumo de alimento $/ \mathrm{N}^{\circ}$ aves por jaula)

- Conversión alimenticia $(\mathrm{CA}=$ Consumo de alimento semanal / masa de huevos semanal).

\section{Análisis Estadístico}

Para el análisis de los datos se utilizó el software R Statistical 4.0 con las librerias ggplot y agricolae. La etapa productiva $\mathrm{y}$ calidad del huevo se analizó mediante el diseño de bloques aleatorios con arreglo factorial $3 \times 3$. El primer factor fue edad al inicio de postura, mientras que el segundo factor fue el peso al inicio de postura. $\mathrm{La}$ comparación de medias se realizó con la prueba Duncan $(p<0.05)$. Las tasas de las variables fueron transformadas con el siguiente método [Ecuación $1\left(\mathrm{Ai}=\operatorname{Arcseno}\left((\mathrm{Yi} / 100)^{1 /}\right.\right.$ $\left.{ }^{2}\right)$ ] (Islam y Al-Shiha, 2018).

\section{Resultados y Discusión}

\section{Comportamiento Productivo}

La masa de huevo, el número de huevos y tasa de postura aumentaron conforme a la edad de la codorniz de postura (Figura 1a,b,c), mientras que la tasa de incubación evidenció una disminución entre la semana 11 y 13 , alcanzando un máximo a la semana 14, y descendiendo sostenidamente hasta la semana 17 (Figura 1d). Por otro lado, la masa de huevo se incrementó desde la semana 9 a la 17, así como el consumo del alimento y la conversión alimenticia (Figura 1e,f,g,h). Jatoi et al. (2013) en codornices japonesas pesadas (300-350 g), medianas (250-300 g) y pequeñas (200-250 g) observaron diferencias en peso corporal, número de huevos, peso del huevo y conversión alimenticia ( $\mathrm{g} / \mathrm{huevo}$ ), pero no encontraron diferencias en la masa de huevo (g/ave). Los resultados del presente estudio difieren en el comportamiento de la masa de huevo, en tanto que la conversión alimenticia no evidencia diferencias significativas en la semana 17 (Cuadro 2), posiblemente debido al menor tamaño de las codornices utilizadas en el presente estudio.

\section{Consumo de Alimento}

El mayor consumo se encontró en aves a los 59 días ( $19.97 \pm 3.00 \mathrm{~g} /$ ave), mientras que para el peso vivo las aves de $150 \mathrm{~g}$ de peso vivo presentaron el mayor consumo (20.73 $\pm 2.57 \mathrm{~g} /$ ave). Por otro lado, el máximo consumo se encontró en aves de 52 días y $150 \mathrm{~g}$ de peso vivo al inicio de postura (Cuadro 2). No se evidenciaron diferencias en 45 y 52 días de edad, tampoco entre los 52 y 59 días de edad; sin embargo, las aves de $150 \mathrm{~g}$ de peso vivo tuvieron un mayor consumo en comparación con las aves de 130 y 140 g (Figura 2).

Se reporta que codornices japonesas desde 30 hasta 168 días de edad con peso inicial de postura de $94.9 \pm 12.5 \mathrm{~g}$, alimentadas con semilla de Moringa oleifera $(19.96 \%$ de proteína y $3011.8 \mathrm{kcal} / \mathrm{kg})$ tuvieron un consumo de $33.23 \mathrm{~g} / \mathrm{día}$, mientras que con dietas con soya integral cocida $(0,5,10$, 15 y $20 \%$ ) tuvieron consumos 24.5 a 25.2 $\mathrm{g} /$ día, sin diferencias en cuanto a la adición de soya (Caetano et al., 2017; Ashour et al., 2020), siendo muy próximos al consumo máximo a los 52 días de edad en el presente estudio $(24.10 \mathrm{~g})$. Por otro lado, con dietas de proteína de 17 a $22 \%$ y energía de 2750 a $3050 \mathrm{kcal} / \mathrm{kg}$ se reportan consumos diarios de 22.2 a 24.9 g (Nery et al., 2010, 2013; Ashour et al., 2020). 

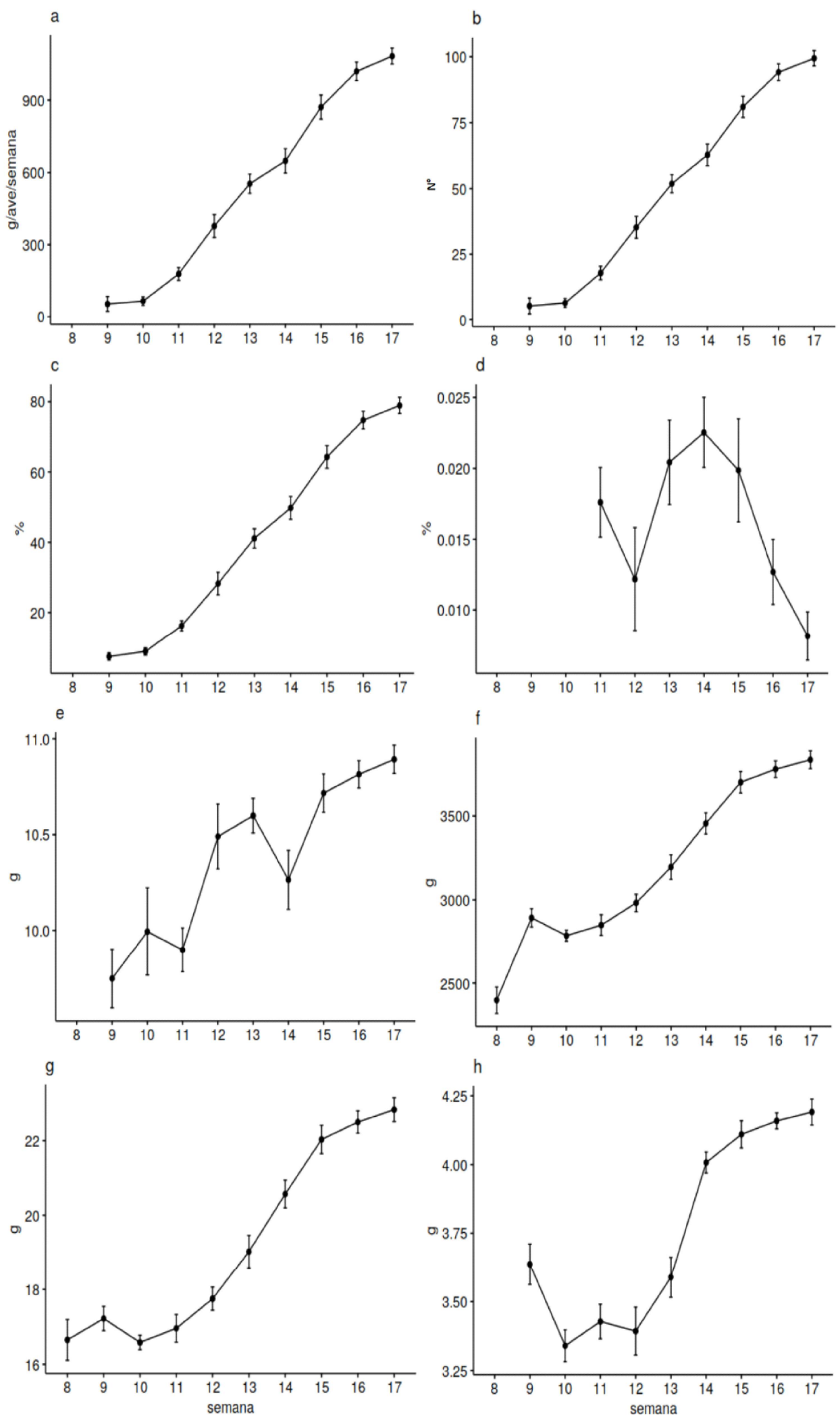

Figura 1. Comportamiento productivo de codornices Coturnix coturnix japonica de tres edades y tres pesos al inicio del empadre. a: masa de huevo, b: número de huevos, c: tasa de postura, d: tasa de incubación, e: Masa de huevo, f: consumo de alimento semanal, $\mathrm{g}$ : consumo de alimento diario y h: conversión alimenticia 


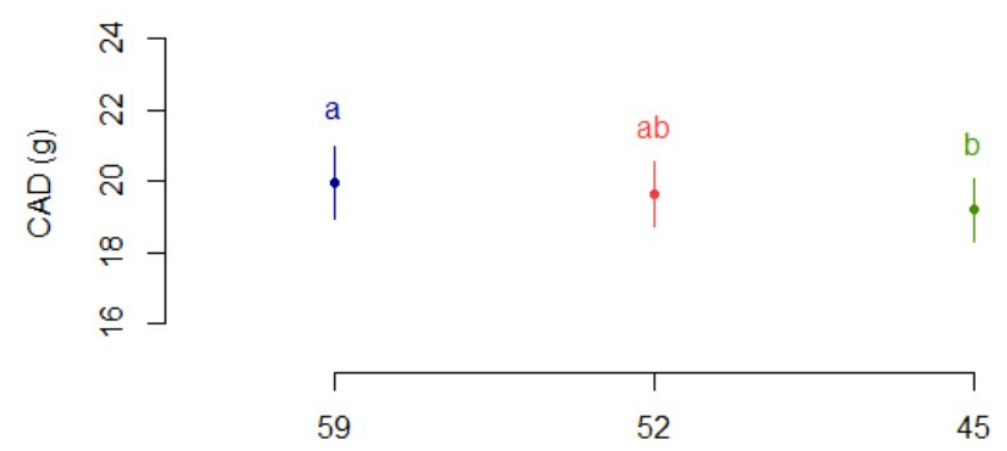

Dias a la postura

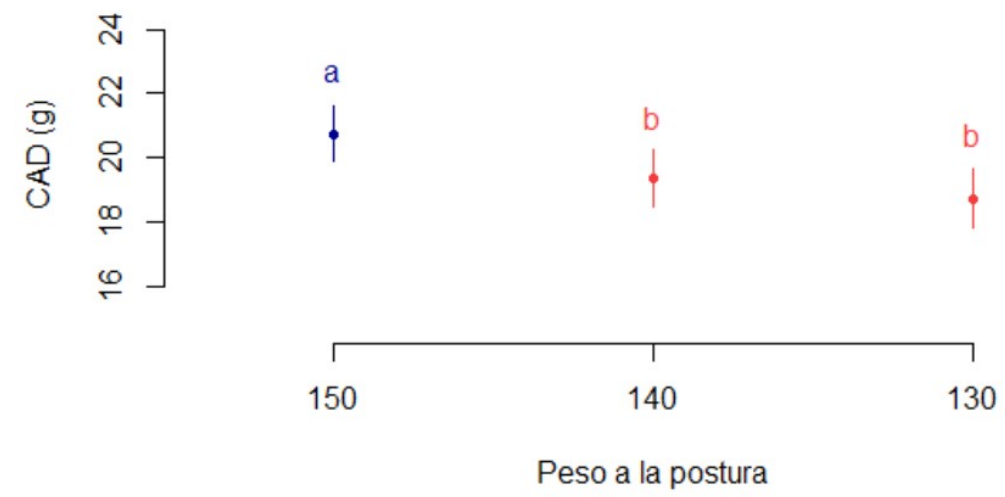

Figura 2. Comparación del consumo diario de alimento (CAD) de codornices Coturnix coturnix japonica según la edad (días) a la postura y peso a la postura (Duncan p-valor $<0.05$ )

Al comparar las edades y los pesos vivos como efectos principales se encontraron evidencias significativas que indicaban que las aves de mayor edad y peso consumieron mayor cantidad de alimento (Figura 2). Es de esperarse que la edad y peso de la codorniz afecten al consumo de alimento diario (Nazligul et al., 2001), así como por la composición genética de la población(Hussain et al., 2016).

\section{Conversión Alimenticia}

La mejor conversión alimenticia se observó en los grupos de codornices de $130 \mathrm{~g}$ con $3.67 \pm 0.42$ y $140 \mathrm{~g}$ con $3.68 \pm 0.35$, siendo significativamente diferentes de la conversión alimenticia observada en codornices de 150 g (3.86 \pm 0.38$)$, en tanto que para la edad no se observaron diferencias significativas (Figura 3). Por otro lado, se reporta que codornices en postura desde 30 hasta 168 días de 


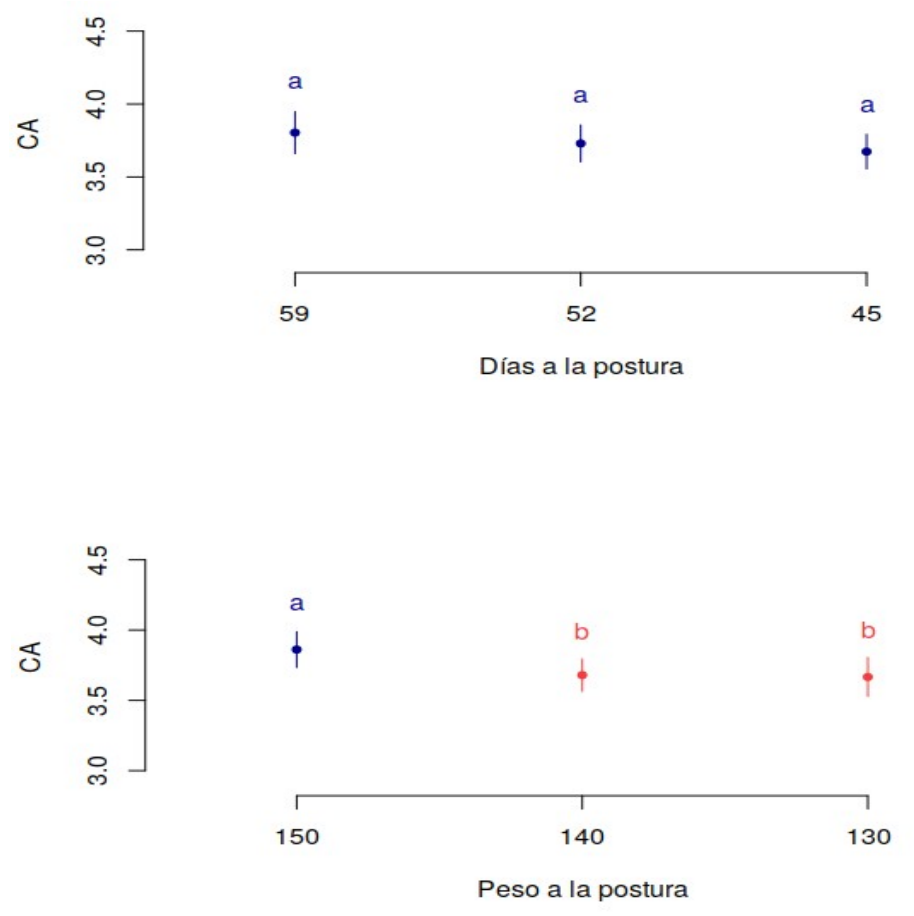

Figura 3. Comparación de la conversión alimenticia de codornices Coturnix coturnix japonica según la edad (días) a la postura y peso a la postura (Duncan $p$-valor $<0.05$ )

edad tuvieron conversiones alimenticias entre 3.47 y 3.95 en dietas de soya cocida con niveles entre y $20 \%$, pero sin diferencias significativas por efecto de los niveles de soya de la dieta (Nery et al., 2010). En este sentido, Da Costa et al. (2017) mencionan un máximo de conversión alimenticia de 13.59 y mínimo de $1.52 \mathrm{~g}$ a los 35 días de edad, en tanto que Ashour et al. (2020) utilizando dietas a base de semilla de M. oleifera encontró una conversión alimenticia de 2.83 , siendo bastante mejor que la reportada en el presente estudio.

\section{Masa de Huevos}

La mayor masa de huevos se manifestó codornices de $150 \mathrm{~g}$ de peso vivo con $10.72 \pm 0.41 \mathrm{~g} /$ día/ave (Cuadro 2), habiendo diferencias significativas con los demás grupos $(\mathrm{p}<0.05)$. Por otro lado, se observa una amplitud de masa de huevos, encontrándose un mínimo de $9.39 \mathrm{~g} /$ día/ave a los 45 días de edad y $130 \mathrm{~g}$ de peso vivo y un máximo de $11.15 \mathrm{~g} /$ día/ave a los $150 \mathrm{~g}$ de peso vivo y 52 días de edad (Cuadro 2). No se encontraron interacciones entre edad a la postura y peso a la postura. Tampoco se observó diferencias estadísticas por influencia de la edad al inicio de la postura (Cuadro 2; Figura 4).

Se reportaron masas de huevo de 10.57 a $11.74 \mathrm{~g} / \mathrm{d}$ ía/ave cuando se alimentaron a las codornices en postura sin y con semilla de M. oleiferia, respectivamente, aunque sin diferencias significativas con el grupo control (Ashour et al., 2020). Permatahati et al. (2019) reportaron que la masa de huevo en codornices de 56 días de edad se incrementó cuando se incluyó en la dieta un insumo proteico no tradicional como Gryllus bimaculatus. De otra parte, de Moraes et al. (2019) indica que el balance electrolítico afecta la calidad del huevo cuando las temperaturas ambientales son altas. 
Cuadro 2. Valores de variables productivas de acuerdo con la edad (días) a la postura y peso vivo $(\mathrm{g})$

\begin{tabular}{|c|c|c|c|c|c|}
\hline Variable & Factor & Nivel & Valor & Min & Max \\
\hline \multirow[t]{6}{*}{ CAD (g) } & Días & 45 & $19.21 \pm 2.63^{b}$ & 16.26 & 23.76 \\
\hline & & 52 & $19.63 \pm 2.74^{\mathrm{ab}}$ & 16.11 & 24.1 \\
\hline & & 59 & $19.97 \pm 3.00^{\mathrm{a}}$ & 15.4 & 23.97 \\
\hline & Peso vivo & 130 & $18.73 \pm 2.81^{b}$ & 15.4 & 22.7 \\
\hline & & 140 & $19.34 \pm 2.63^{b}$ & 16.26 & 22.92 \\
\hline & & 150 & $20.73 \pm 2.57^{\mathrm{a}}$ & 17.62 & 24.1 \\
\hline \multirow[t]{6}{*}{$\mathrm{CA}$} & Días & 45 & $3.67 \pm 0.36^{\mathrm{a}}$ & 3.23 & 4.27 \\
\hline & & 52 & $3.73 \pm 0.38^{\mathrm{a}}$ & 3.26 & 4.32 \\
\hline & & 59 & $3.80 \pm 0.43^{\mathrm{a}}$ & 3.14 & 4.41 \\
\hline & Peso vivo & 130 & $3.67 \pm 0.42^{\mathrm{b}}$ & 3.14 & 4.24 \\
\hline & & 140 & $3.68 \pm 0.35^{\mathrm{b}}$ & 3.23 & 4.19 \\
\hline & & 150 & $3.86 \pm 0.38^{\mathrm{a}}$ & 3.33 & 4.41 \\
\hline \multirow[t]{6}{*}{ MH (g/día/ave) } & Días & 45 & $10.43 \pm 0.54^{\mathrm{a}}$ & 9.39 & 11.14 \\
\hline & & 52 & $10.50 \pm 0.55^{\mathrm{a}}$ & 9.69 & 11.15 \\
\hline & & 59 & $10.46 \pm 0.48^{\mathrm{a}}$ & 9.6 & 10.95 \\
\hline & Peso vivo & 130 & $10.19 \pm 0.47^{\mathrm{c}}$ & 9.39 & 10.72 \\
\hline & & 140 & $10.48 \pm 0.54^{\mathrm{b}}$ & 9.6 & 11.08 \\
\hline & & 150 & $10.72 \pm 0.41^{\mathrm{a}}$ & 10.05 & 11.15 \\
\hline \multirow[t]{6}{*}{ ТP (\%) } & Días & 45 & $43.86 \pm 25.62^{\mathrm{a}}$ & 14.29 & 79.37 \\
\hline & & 52 & $48.51 \pm 27.35^{\mathrm{a}}$ & 15.87 & 88.62 \\
\hline & & 59 & $43.92 \pm 30.92^{\mathrm{a}}$ & 10.02 & 89.42 \\
\hline & Peso & 130 & $42.71 \pm 29.11^{b}$ & 10.02 & 79.37 \\
\hline & & 140 & $42.10 \pm 25.34^{b}$ & 10.65 & 76.72 \\
\hline & & 150 & $51.49 \pm 28.71^{\mathrm{a}}$ & 16.18 & 89.42 \\
\hline
\end{tabular}

CAD: Consumo diario, CA: Conversión alimenticia, MH: Masa de huevo; TP: Tasa de Postura

\section{Tasa de Postura}

Las codornices de $150 \mathrm{~g}$ de peso corporal presentaron una tasa de postura de 51.5 $\pm 28.7 \%$ (Cuadro 2), con diferencias significativas $(\mathrm{p}<0.05)$ entre promedios (Figura 5). Codornices en postura desde 30 hasta 168 días de edad tuvieron tasas de postura entre
61.3 y $66.4 \%$ en dietas de soya cocida con niveles 0 a $20 \%$ sin que se encontrase influencia por los niveles de soya de la dieta (Nery et al., 2010). Por otro lado, la tasa de postura varió de 78.95 a $83.41 \%$, habiendo mejora por efecto de dietas con semilla de M. oleiferia (Ashour et al., 2020). 

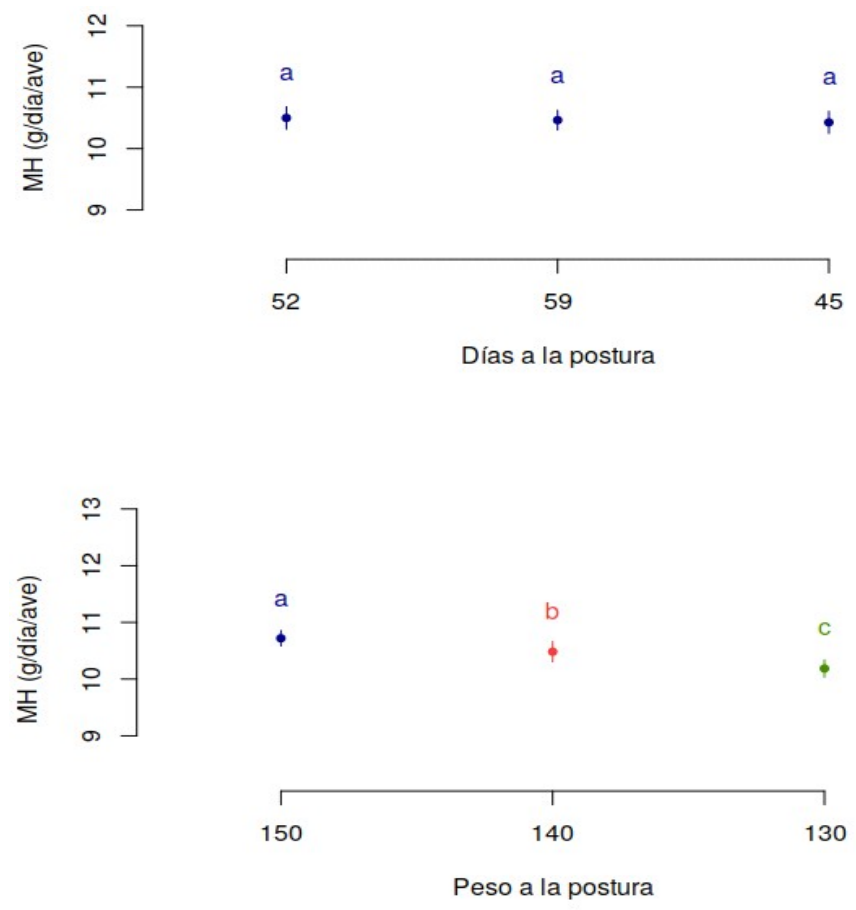

Figura 4. Comparación de la masa de huevo (MH) de codornices Coturnix coturnix japonica según la edad (días) a la postura y peso a la postura (Duncan $\mathrm{p}$-valor $<0.05$ )
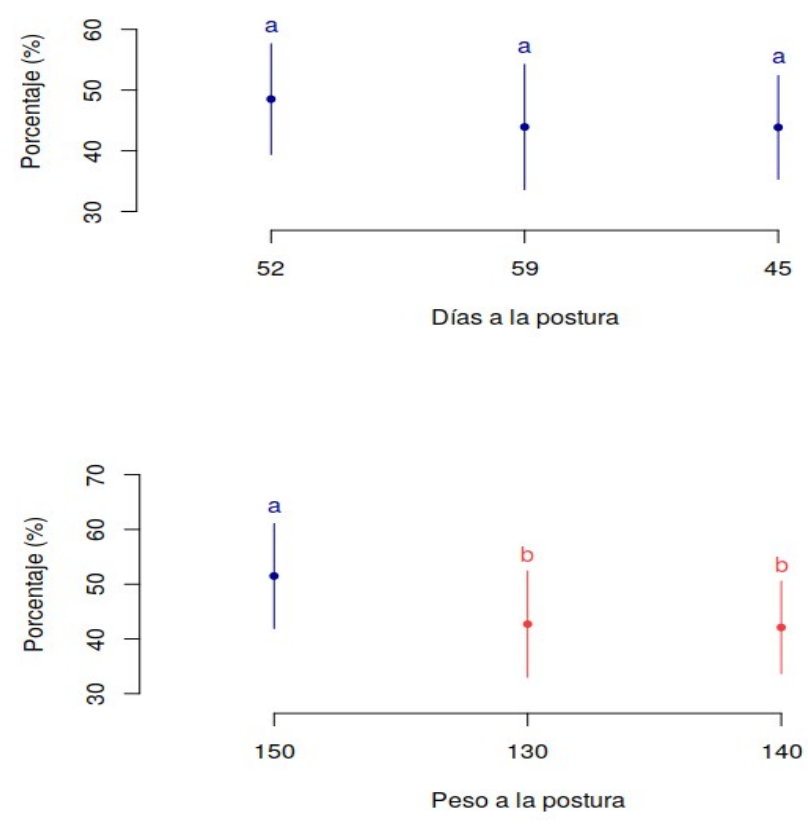

Figura 5. Comparación de la tasa de postura de codornices Coturnix coturnix japonica según la edad (días) a la postura y peso a la postura (Duncan p-valor $<0.05$ ) 


\section{Conclusiones}

- Al finalizar la semana 17 se evidenciaron diferencias significativas en el consumo diario, consumo semanal, masa de huevos, número de huevos y tasa de postura; siendo las codornices que pesaron $150 \mathrm{~g}$ al inicio de la producción de huevos y entre las 52 y 59 semanas las que obtuvieron mejores rendimientos.

- Las codornices con peso vivo al inicio de postura de 130 y $140 \mathrm{~g}$ evidenciaron una mejor conversión alimenticia, aunque sin diferencias significativas.

- La masa de huevo fue directamente proporcional a la edad y peso corporal del animal, siendo el grupo de codornices hembra de $150 \mathrm{~g}$ las que tuvieron mejor respuesta.

\section{Literatura Citada}

1. Ashour EA, El-Kholy MS, Alagawany M, El-Hack MEA, Mohamed LA, Taha AE, Sheikh AIE, et al. 2020. Effect of dietary supplementation with moringa oleifera leaves and/or seeds powder on production, egg characteristics, hatchability and blood chemistry of laying Japanese quails. Sustainability 12: 2463. doi: 10.3390/su12062463

2. Caetano G, Mota R, Silva D, Oliveira H, Viana J, Siqueira O, Freitas $P$, et al. 2017. Bayesian estimation of genetic parameters for individual feed conversion and body weight gain in meat quail. Livest Sci 200: 76-79. doi: 10.1016/j.livsci.2017.04.011

3. Cori ME, Michelangeli C, De Basilio V, Figueroa R, Rivas N. 2014. Solubilidad proteica, contenido de mioglobina, color y $\mathrm{pH}$ de la carne de pollo, gallina y codorniz. Arch Zootec 63: 133-143. doi: 10.4321/S0004-05922014000100013
4. Da Costa Caetano G, Mota RR, da Silva D, Oliveira HR, Viana JMS, Siqueira OHG, Freitas PHF, Silva FF. 2017. Bayesian estimation of genetic parameters for individual feed conversion and body weight gain in meat quail. Livestock Sci 200: 76-79. doi: 10.1016/ j.livsci.2017.04.011

5. Da Cunha RG. 2009. Quail meat-an undiscovered alternative. World Poultry 25: 12-14.

6. de Moraes M, da Rocha C, Moreno T, Surek D, Borges S, Maiorka A. 2019. Effect of different dietary electrolyte balance values at high temperature peaks on performance and egg quality of Japanese quail (Coturnix coturnix japonica). J Appl Poultry Res 28: 12341239.

7. [FAO] Organización de las Naciones Unidas para la Alimentación y la Agricultura. 2013. Revisión del desarrollo avícola. Roma: FAO. 129 p.

8. Galíndez, $R$, De Basilio V, Martínez $G$, Vargas D, Uztariz E, Mejía P. 2009. Evaluación de la fertilidad y eclosión en la codorniz japonesa. Zootec Trop 27: 007-018.

9. Hussain J, Akram M, Javed K, Mahmud A, Mehmood S, Ahmad S, Ahmad F, Jatoi A, Abbas Y, Hussnain F. 2016. Quail breeder's production performance in response to selection for higher three weeks body weight. J Anim Plant Sci 26: 588-593.

10. Islam M, Al-Shiha A. 2018. Foundations of Biostatistics. Springer. $463 \mathrm{p}$.

11. Jatoi A, Sahota A, Akram M, Javed K, Jaspal M, Hussain J, Mirani H, Mehmood S. 2013. Effect of different body weight categories on the productive performance of four close-bred flocks of Japanese quails (Coturnix coturnix japonica). J Anim Plant Sci 23: 7-13.

12. Kuurman WW, Bailey BA, Koops WJ, Grossman M. 2002. Influence of storage days on the distribution for time 
of embryonic mortality during incubation.

Poultry Sci 81:1-8. doi: 10.1093/ps/81.1.1

13. Lukanov H, Pavlova I. 2020. Domestication changes in Japanese quail (Coturnix japonica): a review. Worlds Poult Sci J 76: 787-801. doi: 10.1080/ 00439339.2020 .1823303

14. Nazligul A, Türkyilmaz K, Bardakçiodlu HE. 2001. A study on some production traits and egg quality characteristics of Japanese quail. Turk J Vet Anim Sci 25: 1007-1013.

15. Nery VLH, Corredor LF, Novoa DMT. 2010. Grano de soya integral cocido en la alimentación de codornices. Orinoquia 14: 27-32. doi: 10.22579/20112629.123
16. Nery VLH, Torres-Novoa DM, Ocampo-Durán A. 2013. The effect of protein level on egg-laying Japanese quails. Orinoquia 17: 30-37.

17. Permatahati D, Mutia R, Astuti DA. 2019. Effect of cricket meal (Gryllus bimaculatus) on production and physical quality of Japanese quail egg. Trop Anim Sci J 42: 53-58. doi: 10.5398/tasj.2019.-42.1.53

18. Randall M, Bolla G. 2008. Raising Japanese quail. In: The Poultry Site. https://thepoultrysite.com/articles/raisingjapanese-quail

19. Sahin EH, Sengor E, Yardimci M, Cetingul IS. 2009. Relationship between preincubation egg parameters from old breeder hens, egg hatchability and chick weight. J Anim Vet Adv 8: 115-119. 